\title{
Coronavirus Disease 2019: COSeSco - A Risk Assessment Score to Predict the Risk of Pulmonary Sequelae in COVID-19 Patients
}

\author{
Marina Aiello ${ }^{a}$ Laura Marchi $^{a} \quad$ Luigino Calzetta $^{a} \quad$ Silvia Speroni ${ }^{b}$ \\ Annalisa Frizzelli ${ }^{a}$ Marianna Ghirardini ${ }^{a}$ Valentina Celiberti ${ }^{b}$ \\ Nicola Sverzellatic ${ }^{c}$ Maria Majori ${ }^{d}$ Pier Anselmo Mori ${ }^{d}$ Silvia Ranzieri ${ }^{b}$ \\ Roberta Pisi $^{a}$ Giovanna Pelàe Massimo Corradi $^{b}$ Alfredo Chetta $^{a}$ \\ aDepartment of Medicine and Surgery, Respiratory Disease and Lung Function Unit, University of Parma, Parma, \\ Italy; ${ }^{b}$ Department of Medicine and Surgery, Occupational Medicine Unit, University of Parma, Parma, Italy; \\ 'Department of Medicine and Surgery, Section of Radiology, Unit of Surgical Sciences, University of Parma, Parma,

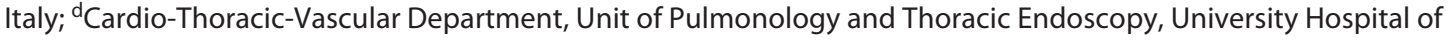

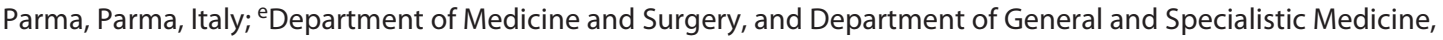 \\ University-Hospital of Parma, University of Parma, Parma, Italy
}

\section{Keywords}

Coronavirus disease 2019 - Lung function · Long COVID ·

Diffusion capacity

\begin{abstract}
Background: The presence of interstitial pneumonia in coronavirus disease 2019 (COVID-19) patients, as diagnosed through laboratory, functional, and radiological data, provides potential predicting factors of pulmonary sequelae. Objectives: The objectives were the creation of a risk assessment score for pulmonary sequelae at high-resolution computed tomography (HRCT) through the assessment of laboratory data, lung function, and radiological changes in patients after the onset of COVID-19 interstitial pneumonia and the identification of predictive factors. Methods: We enrolled 121 subjects hospitalized due to COVID-19 pneumonia in our study. Clinical features, Charlson Comorbidity Index (CCl) score, HRCT score, and blood chemistry data at hospital admission, as well as HRCT score, pulmonary function testing values, exercise capacity by means of a 6-Minute
\end{abstract}

Walk Test (6MWT), and dyspnea perception by the modified Medical Research Council (mMRC) at 4-month follow-up, were all recorded. The variables were elaborated in order to create a predictive model to identify patients at high risk of pulmonary sequelae at HRCT. Results: At the time of followup visit, $63 \%$ of patients had functional abnormality (diffusion lung capacity and/or total lung capacity $<80 \%$ of predicted). Age, BMI, CCl, D-dimer, 6MWT, and mMRC were included in the COVID-19 Sequelae Score (COSeSco, ranging 0-15), which was able to individuate COVID-19 patients with radiologic sequelae (HRCT score $>10 \%$ ) at follow-up. The most revelatory COSeSco value that was found to intercept the highest sensitivity (100\%) and specificity (77\%) was 2 . Conclusion: The COSeSco - comprising age, BMI, comorbidities, D-dimer, walking distance, and dyspnea perception makes it possible to identify particularly at-risk COVID-19 patients who are likely to develop pulmonary sequelae assessed by HRCT.

(c) 2021 S. Karger AG, Basel

Marina Aiello and Laura Marchi contributed equally to this work as first authors. 


\section{Introduction}

In March 2020, the WHO declared a coronavirus disease 2019 (COVID-19) pandemic [1], caused by SARS-CoV-2, a coronavirus featuring a severe acute respiratory syndrome. In its most common presentation, the disease is typified by interstitial pneumonia with a severity ranging from mild to severe, along with the development of acute respiratory distress syndrome, ranging from about $15 \%$ to $30 \%$ [2]. Even after the resolution of the acute phase, most patients could not be characterized as having recovered [3], normally understood as complete restitutio ad integrum of the lung parenchyma. Zhao et al. [4] demonstrated the presence of radiological and physiological abnormalities in a high percentage of COVID-19 patients 3 months after discharge. Furthermore, these abnormalities could be predicted by high levels of D-dimer upon admission [4].

Recent reports suggest that anamnestic (e.g., the presence of comorbidities), laboratory, and functional data may signal interstitial pneumonia during the SARSCoV-2 emergency, thus providing useful indicators for predicting the different levels of severity of the disease and allowing for the assessment of the risk of relevant sequelae [5, 6]. Similarly, Huang et al. [7] observed, in severe cases of SARS-CoV-2 infections, a lung diffusion capacity for carbon monoxide (DLCO) impairment, a more total lung capacity (TLC) decrease, and 6MWT decline, as far as nonsevere cases were concerned.

The aim of the present study was to record clinical lab functional and HRCT data in a large cohort of COVID-19 patients with documented high-resolution computed tomography (HRCT) pneumonia, along with their changes at follow-up, and, subsequently, to identify the risk factors of the HRCT sequelae. Furthermore, the population data were used to determine a predictive score, which we refer to as COSeSco (COVID-19 Sequelae Score), which includes age, BMI, comorbidities, and D-dimer at the time of hospitalization, as well as exercise capacity and dyspnea at follow-up, which allowed us to account for the risk of pulmonary sequelae at HRCT in COVID-19 patients. This score could be useful in the clinical management of COVID-19 patients after discharge and at follow-up.

\section{Materials and Methods}

\section{Collection of Information}

The information for this study was collected from the databases of the "Respiratory Disease and Lung Function Unit," University Hospital of Parma (Italy), from May 2020 to February 2021. As a consequence of the COVID-19 pandemic, the Parma University
Hospital established a post-acute COVID-19 outpatient clinic to follow up with patients who had been previously hospitalized because of a SARS-CoV-2 infection. Participants in the study were patients subsequently referred to a post-acute COVID-19 outpatient clinic who met the following inclusion criteria: adults of both sexes (age $\geq 18$ years) with radiologically and laboratory (nasopharyngeal swabs and/or serological assay) documented diagnosis of COVID-19-related pneumonia and who had been previously hospitalized and discharged. The exclusion criteria were the following: subjects unable to meet the criteria of acceptability and repeatability of functional investigations or who have not signed the informed consent.

Information concerning patient characteristics, such as age, gender, smoking habit, comorbidities via the Charlson Comorbidity Index (CCI) [8], pneumonia with HRCT score (0-100\%) [9], blood chemistry data, and treatment, was collected at the time of hospitalization. In addition, dyspnea with the modified Medical Research Council (mMRC) [10], Pulmonary Function Test (PFT), exercise capacity by means of the 6MWT [11], and HRCT score were collected at a follow-up visit (approximately 4 months after hospital admission) (Table 1).

The study population data were used to determine a predictive score, which, in turn, allowed us to account for the risk of pulmonary sequelae in COVID-19 patients.

\section{Study Design and Statistical Analysis}

We identified the variables that significantly correlated (2-tailed Pearson correlation) with the HRCT score at hospital admission, and then the 2 variables showing the best correlation outcomes were used to characterize the study population in patients with normal lung function or with lung function impairment at followup (patient characterization, step I). After that, we identified the best functional variable (independent variable) that significantly discriminated patients with HRCT sequelae at follow-up. The level of discrimination threshold of functional pulmonary sequelae was then checked for significant variables (dependent variables) with the receiver operating characteristic (ROC) curve to identify specific cutoff (patient characterization, step II). Last, the variables were elaborated to synthesize COSeSco, an easy computable predictive score, in order to identify the patients at higher risk of pulmonary sequelae at HRCT due to COVID-19 at follow-up [12]. The flowchart reporting the study design is shown in Figure 1. The statistical analysis was carried out by using GraphPad Prism 5 software (San Diego, CA, USA), and the level of statistical significance was $p<0.05$.

\section{Results}

\section{Study Population Characteristics}

The characteristics of the study population at hospital admission and at follow-up are reported in Table 1 along with the variables analyzed in the study and the therapies during hospitalization. All patients underwent a followup visit, which was performed approximately 4 months after hospital admission. Seventy-six out of 121 patients (63\%) had functional abnormality (DLCO and/or TLC $<80 \%$ at follow-up). 
Table 1. COVID-19 patient characteristics at hospital admission and follow-up

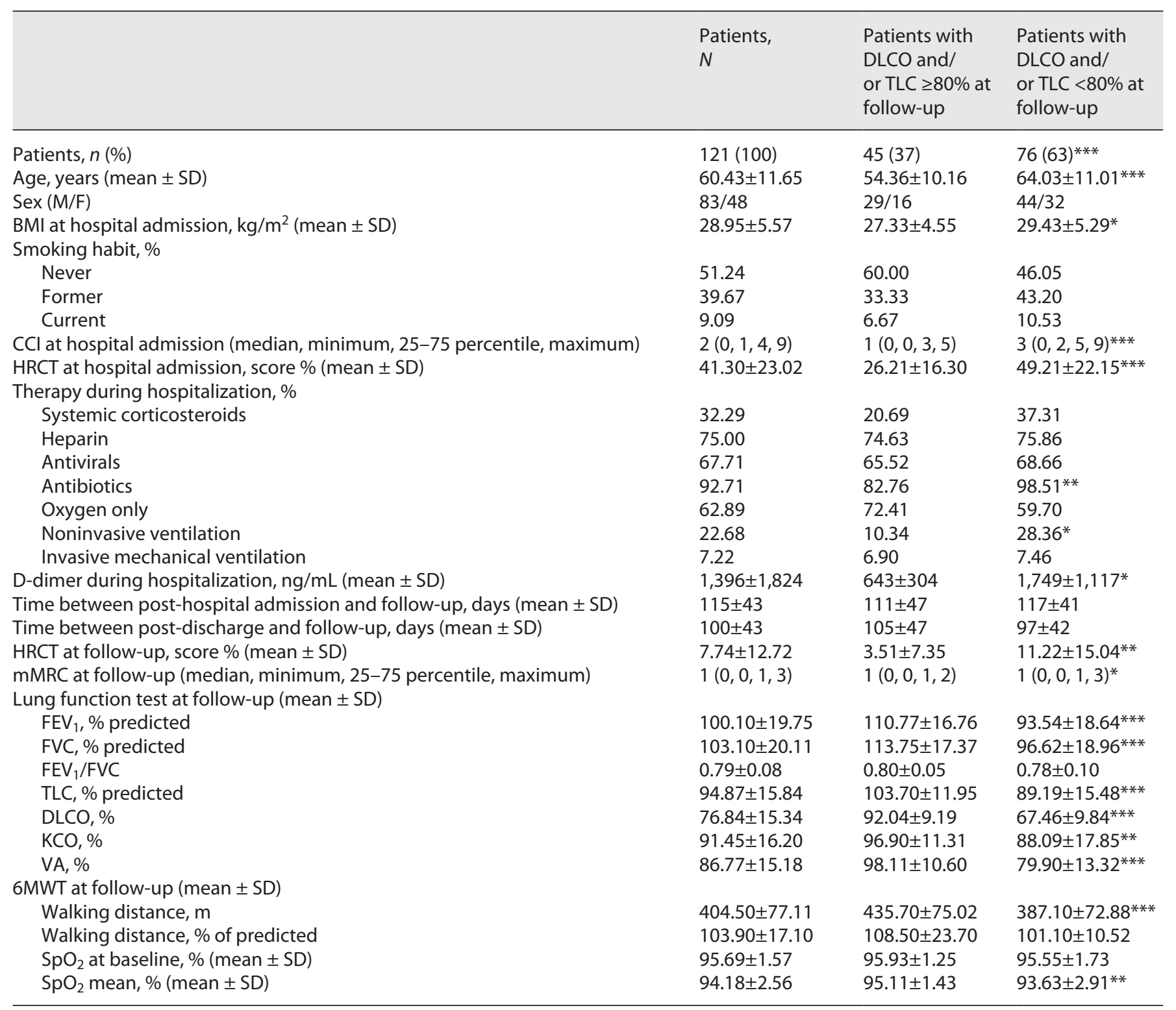

6MWT, 6-Minute Walk Test; BMI, body mass index; COVID-19, coronavirus disease 2019; CCl, Charlson Comorbidity Index; DLCO, lung diffusion capacity for carbon monoxide; $\mathrm{FEV}_{1}$, forced expiratory volume in 1 second; FVC, forced vital capacity; $\mathrm{HRCT}$, high-resolution computed tomography; KCO, DLCO/VA; mMRC, modified Medical Research Council; $n$, number; $m$, meters; $S D$, standard deviation; $S_{20 O}$, peripheral oxygen saturation; TLC, total lung capacity; VA, alveolar volume. ${ }^{*} p<0.05$, ${ }^{* *} p<0.01$, and ${ }^{* * *} p<0.001$ between patients with and without lung function impairment.

\section{Correlation Analysis}

The significant correlation between the investigated variables and HRCT score at hospital admission is reported in online supplementary Figures 1-3 (see www. karger.com/doi/10.1159/000519385 for all online suppl. material). Age, BMI, CCI, forced expiratory volume in 1 second $\left(\mathrm{FEV}_{1}\right)$, forced vital capacity (FVC), DLCO, TLC, alveolar volume (VA), 6MWT distance (m), peripheral oxygen saturation $\left(\mathrm{SpO}_{2}\right)$ at baseline, and the average $\mathrm{SpO}_{2}$ during the $6 \mathrm{MWT}$ significantly $(p<0.05)$ correlated with the HRCT score at hospital admission. No further variables showed significant correlation with the HRCT 
score at hospital admission $(p>0.05)$. Across the significant variables, DLCO and TLC were those characterized by the best correlation outcomes with the HRCT score at hospital admission (Pearson correlation -0.48 , 95\% CI: -0.62 to -0.31 ; and $-0.39,95 \%$ CI: -0.55 to -0.20 , respectively; both $p<0.001$ ).

\section{Patient Characterization}

Patient Characterization (Step I)

By clustering the study population according to nor$\mathrm{mal} / \mathrm{abnormal}$ values $(\geq \mid<80 \%)$ of the functional variables DLCO and/or TLC, we were able to identify 2 different groups of patients characterized by significantly different HRCT scores at hospital admission (difference: $-22.99,95 \%$ CI: -31.68 to $-14.31, p<0.001$ ) and followup (difference: $-7.71,95 \%$ CI: -13.10 to $-2.32, p<0.01$ ), age (difference: $-9.67,95 \% \mathrm{CI}:-13.66$ to $-5.68, p<0.001$ ), BMI (difference: $-2.10,95 \%$ CI: -4.00 to $-0.19, p<0.05$ ), CCI (difference: $-1.87,95 \%$ CI: $-2,59$ to $-1.16, p<0.001$ ), 6MWT distance (m) (difference: 48.57, 95\% CI: 20.8376.31, $p<0.001$ ), mMRC (difference: -0.32 , 95\% CI: -0.61 to $-0.03, p<0.05$ ), and D-dimer (difference: $-1,105$, $95 \%$ CI: $-1,993$ to $-217, p<0.05$ ) outcomes, as shown in Figure 2 and Table 1. Further significant differences were found for $\mathrm{FEV}_{1}$ (\% pred) (difference: $-17.23,95 \% \mathrm{CI}$ : -24.04 to $-10.43, p<0.001$ ), FVC (\% pred) (difference: $-17.13,95 \%$ CI: -24.10 to $-10.17, p<0.001$ ), KCO (difference: $-8.81,95 \%$ CI: -14.70 to $-2.92, p<0.01$ ), VA (difference: $-18.21,95 \%$ CI: -23.13 to $-13.30, p<0.001$ ), and mean $\mathrm{SpO}_{2}$ during the 6MWT at follow-up (difference: $-1.48,95 \% \mathrm{CI}:-2.40$ to $-0.56, p<0.01$ ) (Table 1 ).

Discriminating HRCT Sequelae at Follow-Up with

Functional Variable

Abnormal DLCO value $(<80 \%)$ was effective in significantly $(p<0.05)$ discriminating the study population on the basis of the presence or absence of pulmonary sequelae at follow-up as assessed by the HRCT score (>10\% and $\leq 10 \%$, respectively; online suppl. Fig. $4 a-c)$. Conversely, abnormal TLC values $(<80 \%)$ were not significantly $(p>0.05)$ effective in discriminating patients with and without pulmonary sequelae at follow-up as assessed by the HRCT score (online suppl. Fig. $4 \mathrm{~d}-\mathrm{f}$ ).

The diagnostic effectiveness of DLCO in detecting patients with HRCT score $>10 \%$, as well as the significant $(p$ $<0.05$ ) superiority of DLCO over TLC, was confirmed by the ROC analysis (DLCO area under the curve [AUC]: $0.68,95 \%$ CI: $0.60-0.75, p<0.001$; TLC AUC: $0.52,95 \%$ CI: $0.44-0.60, p>0.05$ ) (Fig. 3a). As expected, the linear analysis of DLCO over the HRCT score at hospital admis-

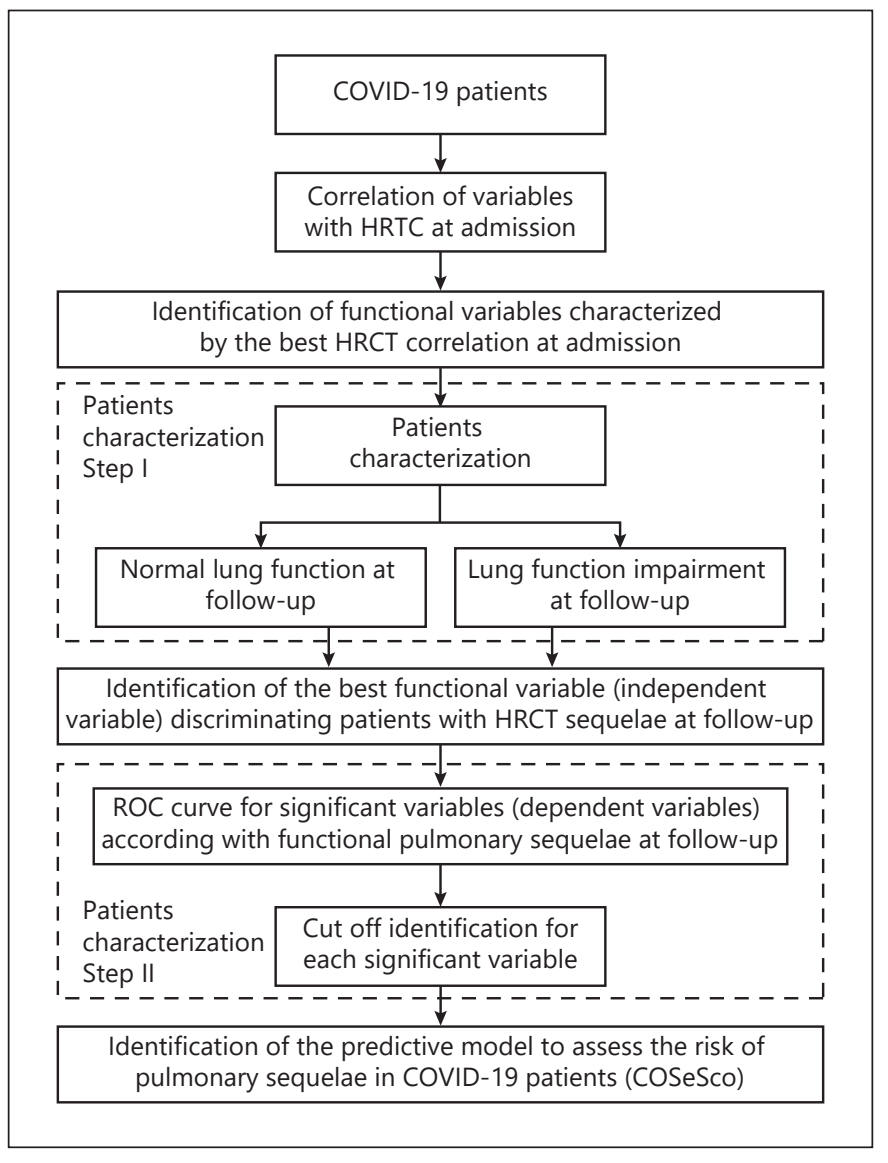

Fig. 1. Flowchart reporting the study design. COSeSco, COVID-19 Sequelae Score; COVID-19, coronavirus disease 2019; HRCT, high-resolution computed tomography; ROC, receiver operating characteristic.

sion and follow-up also reported significant AUC (0.89, 95\% CI: 0.85-0.94, and 0.98, 95\% CI: 0.97-0.99, respectively, both $p<0.001$ ) (Fig. 3b). Moreover, DLCO $<80 \%$ and $\geq 80 \%$ was used as a functional independent variable to discriminate COVID-19 patients with HRCT sequelae (HRCT score $>10 \%$ ) approximately 4 months after hospital admission.

\section{Patient Characterization (Step II)}

The ROC curve analysis shown in Figure 3c-i allows for the identification of the specific characteristics of COVID-19 patients who were still affected, approximately 4 months after hospital admission, by functional sequelae, such as DLCO $<80 \%$ (independent variable) and, accordingly, by radiologic sequelae (HRCT score $>10 \%$ ) as demonstrated above. After considering the specific cutoff of each dependent variable assessed in the ROC analysis (Fig. 3c-i), it was found that these patients were $\geq 65$ years old and char- 


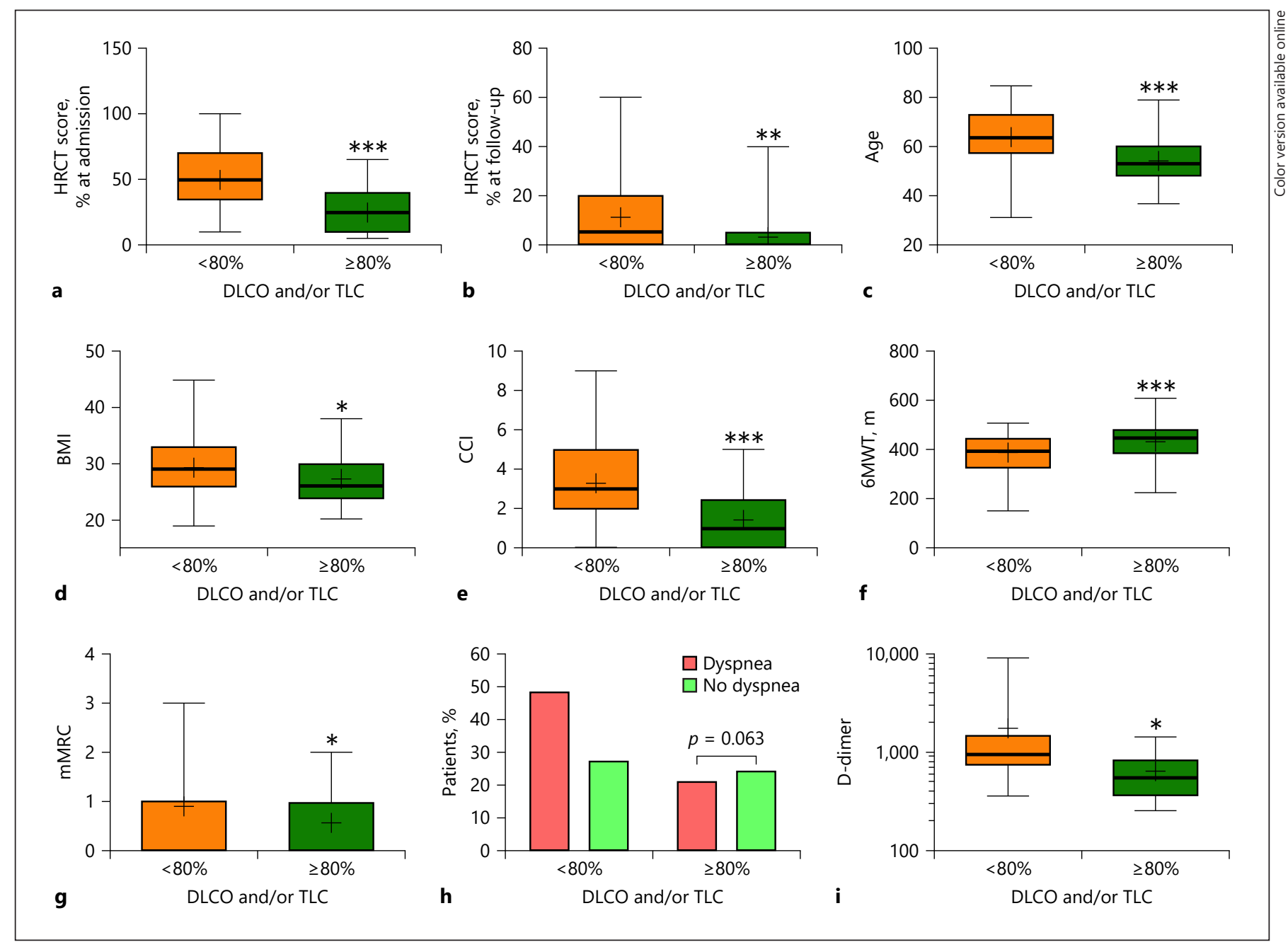

Fig. 2. COVID-19 patients characterized (step I) according to the functional variables DLCO and/or TLC for values $\geq /<80 \%$. Data are reported as median, interquartile range, minimum and maximum $(\mathbf{a}-\mathbf{g}, \mathbf{i})$, and $\%$ of patients (h). Statistical analysis was performed via unpaired Student's $t$ test $(\mathbf{a}-\mathbf{g}, \mathbf{i})$ and $\chi^{2}(\mathbf{h})$. Median, interquartile, and range, the + symbol indicates the mean. ${ }^{*} p<$
$0.05,{ }^{* *} p<0.01$, and ${ }^{* * *} p<0.001 .6 \mathrm{MWT}, 6$-Minute Walk Test; BMI, body mass index; COVID-19, coronavirus disease 2019; CCI, Charlson Comorbidity Index; DLCO, lung diffusion capacity for carbon monoxide; HRCT, high-resolution computed tomography; mMRC, modified Medical Research Council; TLC, total lung capacity. acterized by at least $\mathrm{BMI} \geq 25$, or $\mathrm{CCI} \geq 4$, or $6 \mathrm{MWT}$ (\% pred) $<100 \%$, or $\mathrm{mMRC} \geq 2$, or D-dimer $\geq 500 \mathrm{ng} / \mathrm{mL}$. At this step of the patient characterization, the outcome 6MWT distance was reported as $\%$ of predicted, rather than distance in meters, to make it suitable to identify the specific cutoff for the predictive model described below. The presence of resting dyspnea at follow-up visit was not a prognostic symptom for COVID-19 sequelae after discharge.

\section{Predictive Model}

Data resulting from the ROC analysis of the dependent variables characterizing the patients with functional se- quelae are reported in online supplementary Table 1, and specific scores were assigned to each cutoff according to the level of sensitivity and specificity as follows: age $(<65$ years $=$ score $0, \geq 65$ and $<70$ years $=$ score 1 , and $\geq 70$ years $=$ score 2$),$ BMI $(<25=$ score $0, \geq 25$ and $<30=$ score $1, \geq 30$ and $<35=$ score 2 , and $\geq 35=$ score 3$)$, CCI $(<4=$ score $0,4=$ score $1,5=$ score 2 , and $\geq 6=$ score 3$), 6 \mathrm{MWT}$ (\% pred) $(\geq 100 \%=$ score $0, \geq 90 \%$ and $<100 \%=$ score 1 , $\geq 80 \%$ and $<90 \%=$ score $2, \geq 70 \%$ and $<80 \%=$ score 3 , and $<70 \%=$ score 4$), \operatorname{mMRC}(\leq 1=$ score $0,2=$ score 1 , and $\geq 3$ = score 2$)$, and D-dimer $(<500 \mathrm{ng} / \mathrm{mL}=$ score 0 and $\geq 500 \mathrm{ng} / \mathrm{mL}=$ score 1 ). 


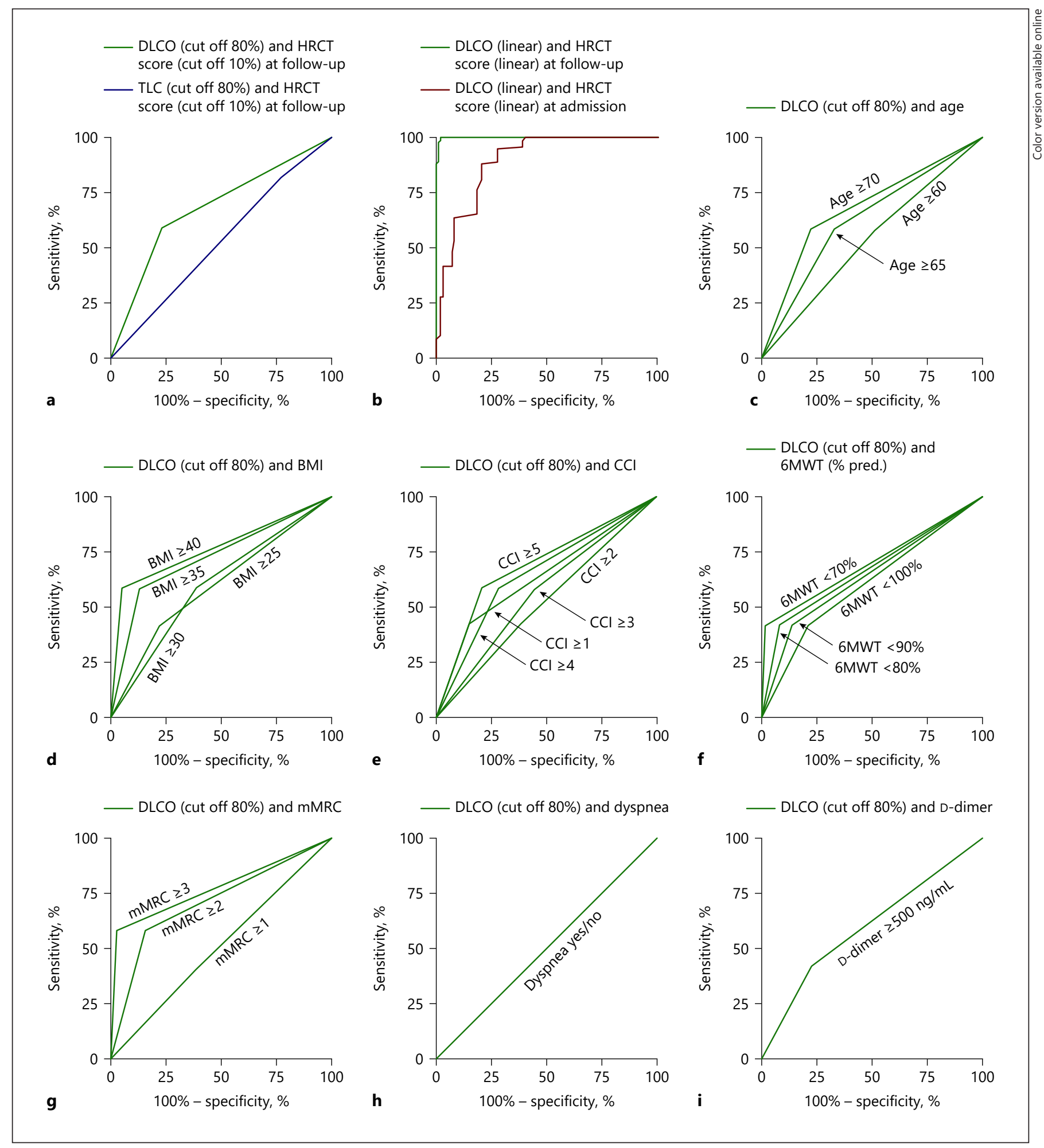

Fig. 3. Discriminating HRCT sequelae at follow-up via functional variables (DLCO and TLC) (a), linear analysis of DLCO over the HRCT score at hospital admission and follow-up (b), and COVID-19 patient characterization (step II) according to the ROC analysis (c-i). 6MWT, 6-Minute Walk Test; BMI, body mass index;
COVID-19, coronavirus disease 2019; CCI, Charlson Comorbidity Index; DLCO, lung diffusion capacity for carbon monoxide; HRCT, high-resolution computed tomography; mMRC, modified Medical Research Council; ROC, receiver operating characteristic; TLC, total lung capacity. 
Next, the scores were further analyzed and pooled in order to synthesize the COSeSco model according to the following equation: $\mathrm{COSeSco}=$ age $($ score $)+\mathrm{BMI}$ (score) $+\mathrm{CCI}$ (score) $+6 \mathrm{MWT}$ (\% pred) (score) + mMRC (score) $+\mathrm{D}$-dimer (score). The sum of the scores assigned the cutoff of each dependent variable and resulted in COSeSco values ranging from 0 to 15 . The ROC analysis indicated that COSeSco was significantly $(p<0.001)$ effective in discriminating COVID-19 patients with radiologic sequelae (HRCT score $>10 \%$ ) at follow-up. The best model for discerning the greatest overall sensitivity and specificity is that for COSeSco values of 2 (sensitivity: 100\%, 95\% CI: $67.54 \%-86.44 \%$; specificity $76.86,95 \%$ CI: $68.32 \%-$ $84.04 \%)$.

\section{Discussion}

In this study, we analyzed clinical, functional, and radiological data from 121 patients with a confirmed diagnosis of COVID-19-related pneumonia. Seventy-six of them $(63 \%)$ at the follow-up visit, performed about 4 months after hospital admission, showed radiological and lung function impairment in terms of diffusing lung capacity and lung volumes with an average HRCT score of $11.22 \%$. Moreover, by clustering the study population according to the normal/abnormal values of DLCO $(\geq /<80 \%)$, we found that patients with lung functional impairment after discharge had certain specific features, and after considering a specific cutoff of such variables, we were able to conclude that these patients were $\geq 65$ years old and characterized by at least BMI $\geq 25$, or CCI $\geq 4$, or $6 \mathrm{MWT}$ ( $\%$ pred) $<100 \%$, or $\mathrm{mMRC} \geq 2$, or $\mathrm{D}$-dimer $\geq 500 \mathrm{ng} / \mathrm{mL}$. These variables were further analyzed and pooled in order to calculate the COSeSco, ranging from 0 to 15 , which was able to significantly discriminate COVID-19 patients with radiologic sequelae (HRCT score $>10 \%$ ) at follow-up. The best COSeSco model for discerning the highest sensitivity (100\%) and specificity (77\%) was 2.

Available data from previous outbreaks sustained by other coronaviruses provide remarkable information about the prevalence of respiratory sequelae. Between $20 \%$ and $60 \%$ of SARS and MERS survivors reported persistent functional alterations and radiological abnormalities consistent with pulmonary fibrosis [13-15]. Based on these previous reports, it is conceivable that respiratory complications may represent an important consequence of COVID-19 [16, 17]. Furthermore, post-acute COVID-19, the so-called long COVID, seems to be a multi- system disease, one which may occur after even a mild acute illness [18].

A recently published study evaluated long-term health consequences 6 months after symptom onset [19]. This study reported that the percentage of patients with abnormal DLCO or TLC values ( $<80 \%$ of predicted) during follow-up was high $(22-56 \%$ and $11 \%-35 \%$, respectively, across different severity scales of disease at the acute phase). Patients with impairment in lung diffusion capacity presented a higher CT score at follow-up. The results of our study are in line with these previous findings.

It is of note that previous studies have not allowed researchers to establish with certainty if gender, age, and comorbidities significantly affect the risk of developing long-term consequences in COVID-19 patients [20], although these variables are correlated with the degree of severity of the disease [21]. Zhao et al. [4] found that lung function changes after 3 months after discharge were more common among patients with high markers of inflammation or impaired coagulation at admission, consisting in high values of $\mathrm{D}$-dimer. Moreover, patients with an abnormal CT score at follow-up were significantly older in comparison with patients with normal CT. However, patients with normal lung function did not differ in age with respect to patients with abnormal lung function. Conversely, Huang et al. [19] showed that age was positively associated with lung diffusion impairment and negatively associated with the percentage of CT score change. In the present study, we further confirmed the significant relationship between age and lung diffusion capacity and HRCT score.

The presence of abnormal lung function (mainly due to a decrease in the DLCO values) after discharge is reported in most follow-up studies [22, 23]. A recent study reported a greater 6MWT decline in terms of walking distance (meters and predicted values) during the followup visits in severe patients compared with nonsevere ones [7]. The severe group also showed a higher incidence of DLCO impairment [7], thus confirming the close relationship between DLCO and 6MWT. In the present study, we provided the evidence that patients with a DLCO of $<80 \%$ of predicted at a follow-up visit had a high likelihood of having a walking distance $<100 \%$ of predicted. In addition, we found that this group of patients had a significant reduction in the KCO value, a finding which suggests an injury of the alveolar-capillary barrier. Interestingly, in this study, unlike DLCO, TLC values were not significantly effective in discriminating patients with and without pulmonary sequelae at followup as assessed by the HRCT score. It is reasonable to as- 
sume that pneumonia induced by SARS-CoV-2 infection, rather than by a restrictive dysfunctional defect, generally leads to an impairment of gas exchanges and a tendency toward ventilatory inhomogeneity, represented by a reduction in VA.

In this study, we developed a risk score to predict the development of functional and radiological sequelae among hospitalized COVID-19-infected patients. The performance of this risk score was satisfactory with an accuracy based on AUC of 0.94. The 6 dependent variables, characterizing the patients with the functional sequelae required for the calculation of the risk of developing critical illness, are readily available. To our knowledge, CO$\mathrm{SeSco}$ is the first scoring rubric for predicting long-term radiologic sequelae among COVID-19 patients. Some risk factors identified in the present study were also applied in prediction models for severity of disease [24] or for mortality of COVID-19 patients [25].

There are some limitations in this study. First, the lack of baseline data of pulmonary function and 6MWT makes it difficult to attribute the impaired pulmonary function to COVID-19 with certainty, in that the baseline lung function of the study group may not have been normal due to concomitant chronic respiratory diseases. Second, the absence of a control cohort makes it necessary to validate COSeSco on an external population before putting it into common use. Moreover, due to the intrinsic nature of the study and sample size, it was not possible to adequately stratify the risk of predicted sequelae according to low, intermediate, and high levels of risk. In any case, results reported in Figure 3 and online supplementary Table 1 clearly show a ranking of risk between specific variables (i.e., age, BMI, CCI, 6MWT, and $\mathrm{mMRC}$ ) and predicted sequelae according to ROC analysis that, along with data on $\mathrm{D}$-dimer, made it possible to develop the COSeSco. Another limitation of the present study is that it involves a single center, and, therefore, further multicenter studies are required to confirm our results.

\section{Conclusion}

This study demonstrated that functional abnormality may occur in a high proportion of COVID-19 patients approximately 4 months after hospital admission due to interstitial pneumonia. The COSeSco, including age, BMI, comorbidities, D-dimer, walking distance, and dyspnea perception, makes it possible to identify particularly at-risk COVID-19 patients who are likely to develop pulmonary sequelae assessed by HRCT at the 4-month follow-up. The COSeSco has the potential to provide individualized health care strategies by offering the best possible care to patients. However, further investigation will be required to evaluate long-term pulmonary sequelae.

\section{Acknowledgment}

The authors gratefully acknowledge Ms. Karen E. Brothers for reviewing the text.

\section{Statement of Ethics}

This study was approved by the Hospital Ethics Committee of North Emilia Area (Approval No. 34570, dated September 14, 2020) in agreement with the Declaration of Helsinki. This research was carried out in accordance with the approved guidelines. Written informed consent was obtained from all participants before inclusion. Data were recorded with the written consent of the patients, lawfully stored, managed according to privacy rules, and with all identifying details blinded.

\section{Conflict of Interest Statement}

The authors have no conflicts of interest to declare.

\section{Funding Sources}

There was no funding source for this study.

\section{Author Contributions}

C.A. conceived and designed the study. A.M. and M.L. had full access to all of the data and took responsibility for the integrity of the data and the accuracy of the data analysis. M.L., S.N., M.M., M.P.A., C.M., P.G., F.A., S.S., C.V., R.S., G.M., and P.R. contributed to the acquisition and collection of data. C.L. analyzed and contributed to the statistical analysis of the data. C.L., C.A., A.M., and M.L. prepared and reviewed the manuscript. All other authors provided critical feedback and approved the final draft.

\section{Data Availability Statement}

All data generated or analyzed during this study are included in this article and its online supplementary material. Further enquiries can be directed to the corresponding author. 


\section{References}

1 WHO (World Health Organization). WHO Director-General's opening remarks at the media briefing on COVID-19 - 11 March 2020. [Internet]. WHO (World Health Organization); 2020. Available from: https://www. who.int/director-general/speeches/detail/ who-director-general-s-opening-remarks-atthe - media - briefing-o n - covid 19---11-march-2020.

2 Critical care committee of Chinese Association of Chest Physician; Respiratory and critical care group of Chinese Thoracic Society; Respiratory care group of Chinese Thoracic Society. Conventional respiratory support therapy for severe acute respiratory infections (SARI): clinical indications and nosocomial infection prevention and control. Zhonghua Jie He He Hu Xi Za Zhi. 2020 Feb 16;43:E015.

3 Mandal S, Barnett J, Brill SE, Brown JS, Denneny EK, Hare SS, et al. "Long-COVID": a cross-sectional study of persisting symptoms, biomarker and imaging abnormalities following hospitalisation for COVID-19. Thorax. 2021 Apr;76(4):396-8.

4 Zhao YM, Shang YM, Song WB, Li QQ, Xie $\mathrm{H}, \mathrm{Xu} \mathrm{QF}$, et al. Follow-up study of the pulmonary function and related physiological characteristics of COVID-19 survivors three months after recovery. EClinicalMedicine. 2020;25:100463.

5 Wu C, Chen X, Cai Y, Xia J, Zhou X, Xu S, et al. Risk factors associated with acute respiratory distress syndrome and death in patients with coronavirus disease 2019 pneumonia in Wuhan, China. JAMA Intern Med. 2020; 180(7):934

6 Epidemiology Working Group for NCIP Epidemic Response, Chinese Center for Disease Control and Prevention. The epidemiological characteristics of an outbreak of 2019 novel coronavirus diseases (COVID-19) in China. Zhonghua Liu Xing Bing Xue Za Zhi. 2020; 41(2):145-51

7 Huang Y, Tan C, Wu J, Chen M, Wang Z, Luo $\mathrm{L}$, et al. Impact of coronavirus disease 2019 on pulmonary function in early convalescence phase. Respir Res. 2020;21(1):163.
8 Tuty Kuswardhani RA, Henrina J, Pranata R, Anthonius Lim M, Lawrensia S, Suastika K. Charlson comorbidity index and a composite of poor outcomes in COVID-19 patients: a systematic review and meta-analysis. Diabetes Metab Syndr. 2020;14(6):2103.

9 Rogliani P, Calzetta L, Coppola A, Puxeddu E, Sergiacomi G, D'Amato D, et al. Are there pulmonary sequelae in patients recovering from COVID-19? Respir Res. 2020;21(1):286.

10 Carvalho-Schneider C, Laurent E, Lemaignen A, Beaufils E, Bourbao-Tournois C, Laribi S, et al. Follow-up of adults with noncritical COVID-19 two months after symptom onset. Clin Microbiol Infect. 2021 Feb;27(2):25863.

11 Pandit R, Vaity C, Mulakavalupil B, Matthew A, Sabnis K, Joshi S. Unmasking hypoxia in COVID 19 - six minute walk test. J Assoc Physicians India. 2020;68(9):50-1.

12 Cazzola M, Calzetta L, Matera MG, Muscoli S, Rogliani P, Romeo F. Chronic obstructive pulmonary disease and coronary disease: COPDCoRi, a simple and effective algorithm for predicting the risk of coronary artery disease in COPD patients. Respir Med. 2015; 109(8):1019-25.

13 Hui DS, Joynt GM, Wong KT, Gomersall CD, Li TS, Antonio G, et al. Impact of severe acute respiratory syndrome (SARS) on pulmonary function, functional capacity and quality of life in a cohort of survivors. Thorax. 2005; 60(5):401-9.

14 Das KM, Lee EY, Singh R, Enani MA, Al Dossari K, Van Gorkom K, et al. Follow-up chest radiographic findings in patients with MERS$\mathrm{CoV}$ after recovery. Indian J Radiol Imaging. 2017;27(3):342.

15 Antonio GE, Wong KT, Hui DS, Wu A, Lee $\mathrm{N}$, Yuen EH, et al. Thin-section CT in patients with severe acute respiratory syndrome following hospital discharge: preliminary experience. Radiology. 2003;228(3):810-5.

16 Shi H, Han X, Jiang N, Cao Y, Alwalid O, Gu $\mathrm{J}$, et al. Radiological findings from 81 patients with COVID-19 pneumonia in Wuhan, China: a descriptive study. Lancet Infect Dis. 2020;20(4):425-34.
17 Zhang T, Sun LX, Feng RE. (Comparison of clinical and pathological features between severe acute respiratory syndrome and coronavirus disease 2019). Zhonghua Jie $\mathrm{He} \mathrm{He} \mathrm{Hu}$ Xi Za Zhi. 2020;43(6):496-502.

18 Greenhalgh T, Knight M, A'Court C, Buxton $\mathrm{M}$, Husain L. Management of post-acute covid-19 in primary care. BMJ. 2020;370: m3026.

19 Huang C, Huang L, Wang Y, Li X, Ren L, Gu $\mathrm{X}$, et al. 6-month consequences of COVID-19 in patients discharged from hospital: a cohort study. Lancet. 2021;397(10270):220-32.

20 Landi F, Gremese E, Bernabei R, Fantoni M, Gasbarrini A, Settanni CR, et al. Post-COVID-19 global health strategies: the need for an interdisciplinary approach. Aging Clin Exp Res. 2020;32(8):1613.

21 Haut Conseil de la Santé Publique (HCSP). Provisional statement: Recommendations on prevention and management of Covid-19 in patients at risk of severe forms. HCSP; 2020.

22 Torres-Castro R, Vasconcello-Castillo L, Alsina-Restoy X, Solis-Navarro L, Burgos F, Puppo $\mathrm{H}$, et al. Respiratory function in patients post-infection by COVID-19: a systematic review and meta-analysis. Pulmonology. 2021;27(4):328-37.

23 Qin W, Chen S, Zhang Y, Dong F, Zhang Z, $\mathrm{Hu} \mathrm{B}$, et al. Diffusion capacity abnormalities for carbon monoxide in patients with $\mathrm{CO}$ VID-19 at three-month follow-up. Eur Respir J. 2021 Jul 22;58(1):2003677.

24 Gong J, Ou J, Qiu X, Jie Y, Chen Y, Yuan L, et al. A tool to early predict severe corona virus disease 2019 (COVID-19): a multicenter study using the risk nomogram in Wuhan and Guangdong, China. Clin Infect Dis. 2020 Jul 28;71(15):833-40.

25 Wang K, Zuo P, Liu Y, Zhang M, Zhao X, Xie $S$, et al. Clinical and laboratory predictors of in-hospital mortality in patients with coronavirus disease-2019: a Cohort Study in Wuhan, China. Clin Infect Dis. 2020;71(16):2079. 